\title{
THE SEMANTIC VIEW, EMPIRICAL ADEQUACY, AND APPLICATION
}

\author{
MaUricio SuÁrez \\ Departamento de Lógica y Filosofía de la Ciencia \\ Facultad de Filosofía \\ Universidad Complutense de Madrid \\ msuarez@filos.ucm.es
}

\begin{abstract}
SUMMARY: It is widely accepted in contemporary philosophy of science that the domain of application of a theory is typically larger than its explanatory covering power: theories can be applied to phenomena that they do not explain. I argue for an analogous thesis regarding the notion of empirical adequacy. A theory's domain of application is typically larger than its domain of empirical adequacy: theories are often applied to phenomena from which they receive no empirical confirmation.
\end{abstract}

KEY WORDS: scientific epistemology, empirical adequacy, semantic conception, scientific representation

RESUMEN: Existe en la filosofía de la ciencia actual un amplio consenso al afirmar que el dominio de aplicación de una teoría científica es en general mucho más extenso que su dominio explicativo: las teorías científicas pueden ser aplicadas a fenómenos que no son capaces de explicar. En este artículo defiendo una tesis análoga con respecto a la noción de adecuación empírica. El dominio de aplicación de las teorías científicas es en general mucho más amplio que su dominio de adecuación empírica: las teorías a menudo se aplican a fenómenos que no proporcionan confirmación empírica a su favor.

PALABRAS CLAVE: epistemología de la ciencia, adecuación empírica, concepción semántica, representación científica

\section{Introduction: A Map of the Arguments}

In this paper I argue for the following claim: the domain of possible applications of a scientific theory is typically much larger than its domain of empirical adequacy. In other words, a theory can be applied to many phenomena that render it no empirical confirmation. Application and confirmation are distinct notions that in general must be kept separate. I argue for this claim within a broadly construed "semantic" conception of scientific theories, since I accept that the semantic conception provides a neater characterisation of the empirical content of a theory than the "syntactic" conception. My conjecture is, however, that this claim does not essentially depend on the semantic conception, but could similarly be argued in the context of a syntactic account of scientific theories and their empirical adequacy. 
The structure of the paper is as follows. In section 2, I find a possible historical source for the conflation of the notions of confirmation and application in the work of Pierre Duhem, the French historian and philosopher of physics. I find that Duhem himself had distinguished very clearly between these two notions in his general account of physical theory; yet he went on to find them coincidental in practice in the history of astronomy. In sections 3 to 5 I work out the details of a notion of empirical adequacy within the semantic conception of scientific theories, leaning heavily on work by Bas van Fraassen, Michael Friedman and Mathias Kaiser. In particular, in section 3, I review arguments in favour of a semantic conception account of the empirical content of a theory. In section 4, I distinguish between the two conceptions of empirical adequacy that have been developed within the semantic conception, in terms respectively of the "embedding" and the "reduction" of phenomenological structures within theoretical structures. I also show that reduction is logically stronger than embedding. A problematic feature of these accounts is the assumption that phenomena possess structures, so in section 5, I review attempts to endow phenomena with structure and argue in favour of Mathias Kaiser's very liberal account. Then, finally, in section 6, I contend, with recourse to an example from the history of superconductivity, that the domain of application of a scientific theory does not coincide with its domain of empirical adequacy, whether understood as embedding or as reduction.

\section{Application, Explanation, Confirmation}

In The Aim and Structure of Physical Theory Pierre Duhem argued that the aim of physics is "to save the phenomena". The physicist's task is to construct physical theories that account for phenomena, in the following two ways. First, theories provide scientists with a Machian economy of thought that enables them to hold in mind a number of empirical regularities all at once:

Instead of a great number of laws offering themselves as independent of one another, each having to be learnt and remembered on its own account, physical theory substitutes a very small number of propositions, viz. fundamental hypotheses. [...] Such condensing of a multitude of laws into a smaller number of principles affords enormous relief to the human mind, which might not be able without such an artifice to store up the new wealth it acquires daily. (Duhem 1954, p. 21) 
Secondly, theories contain only the most abstract principles that can classify and impose structure upon the diversity of natural phenomena:

Experimental physics supplies us with laws all lumped together and, so to speak, on the same plane, without partitioning them into groups of laws united by a kind of family tie. [...] On the other hand theory, by developing the numerous ramifications of the deductive reasoning which connects principles to experimental laws, establishes an order and a classification among these laws. [...] Theory gives, so to speak, the table of contents and the chapter headings under which the science to be studied will be methodologically divided, and it indicates the laws which are to be arranged under each of these chapters. (Duhem 1954, pp. 23-24)

The physical laws that theoretical hypotheses classify are not themselves empirical regularities, but rather inductive generalizations of empirical regularities. Observed regularities necessarily have only a finite number of instances - as they are constituted by a collection of concrete facts, normally relating to past spatio-temporal coincidences between particular kinds of events - while laws have a potentially infinite number of instances. Hence the observed regularities do not fix the physical laws. In modern philosophical jargon, physical laws are underdetermined by phenomena. Similarly, for Duhem, physical theory is underdetermined by the set of physical laws. As a matter of principle there will be several hypotheses that can equally well classify the set of physical laws, and equally well account for the phenomena.

According to Duhem, the truth of a theory can only manifest itself in the theory's capacity to account for the phenomena: "agreement with experience is the sole criterion of truth for a physical theory" (Duhem 1954, p. 21). However, two empirically equivalent theories may postulate radically different properties of the entities and processes that underlie the phenomena. (The Ptolemaic system of the world, for instance, postulates that the earth is static at the centre of the Universe, while in the Copernican system, the earth follows a perfect circular motion around the sun.) This yields the well-known sceptical argument from underdetermination: How are we to choose, among all these empirically equivalent possibilities, the one and only true theory? If the truth of theories manifests itself only in their ability to save the phenomena then it is not possible to select the 
true theory from among the set of empirically indistinguishable hypotheses. The assertion that one of them is true becomes an empty metaphysical claim, devoid of empirical content. ${ }^{1}$

The only necessary requirement for accepting a theory is that it must save the phenomena. But how exactly is a theory supposed to "save the phenomena"? How can physicists know when the theory has achieved its aim to "save the phenomena"? And how much of the phenomena is a theory supposed to "save"? In a collection of papers originally published in 1908, and now available under the title $T o$ Save the Phenomena, Duhem suggests that astronomy, in the tradition of Eudoxus and Ptolemy will provide the model: theories save the phenomena in just the same fashion astronomical hypotheses describe the observed motions of the objects in the heavens. ${ }^{2}$

The observable predictions of the theory are found by deduction from first premises expressing nomological relations between physical quantities, together with boundary conditions and a number of auxiliary assumptions about the workings of the instruments, etc. These predictions must be borne out if the theory is to "save the phenomena". This hypothetico-deductive methodology is of course not in contradiction with Duhem's belief in underdetermination. An experimental contradiction of a theoretical prediction does not necessarily result in a refutation of the theory, as the underdetermination argument still applies in its holistic form:

${ }^{1}$ This doctrine is essentially of scholastic origin; Duhem (1954, p. 41) approvingly quotes Thomas Aquinas: "Astronomers have tried in diverse ways to explain this motion [of the planets]. But it is not necessary that the hypotheses they have imagined be true, for it may be that the appearances the stars present might be due to some mode of motion yet unknown by men."

${ }^{2}$ In To Save the Phenomena Duhem describes two competing astronomical traditions. In the tradition of Eudoxus and Ptolemy celestial phenomena are "saved" if the motions of the objects in the heavens can be calculated and predicted. In the tradition of Aristotle and Posidonius some further conditions must be satisfied: hypotheses about the motions of the objects in the heavens must be based upon the solid principles of physics. The Aristotelian tradition was lost for centuries to the Christian world, preserved only in the Arab writings of Averroes and AlBitrogi, translated into Latin only late in the Middle Ages in the court of Alfonso X of Castille; it was adopted during the Renaissance by the Italian Averroists of the School of Padua and, according to Duhem, inherited by Copernicans and Inquisitors alike (although with obviously competing interpretations) during the Galilean trials. The former tradition, which Duhem favours, began with Eudoxus and Ptolemy; it was pursued in Christianity by the scholastics of the University of Paris during the thirteenth and fourteenth centuries and their followers in German-speaking countries thereafter, and eventually gave rise to the conciliatory and ecumenical views of Osiander and Bellarmino. 
The physicist can never subject an isolated hypothesis to experimental test, but only a whole group of hypotheses; when the experiment is in disagreement with his predictions, what he learns is that at least one of the hypotheses constituting this group is unacceptable and ought to be modified; but the experiment does not designate which one should be changed. (Duhem 1954, p. 187)

What is interesting about Duhem's later historical work is that in the astronomical model both the application and the testing of astronomical hypotheses follow the hypothetico-deductive method. In order to test a hypothesis about the constitution of the heavens, we look for a derivation from the hypothesis of a sequence of positions of a planet, given the appropriate boundary conditions, and we test that sequence by direct observation. Equally a model for the motion of a planet is a sequence of positions deduced from a hypothesis, in just the same manner.

However the distinction between confirmation and application is coherent, and it is important. Duhem himself was well aware of the distinction, and he carefully differentiated between what he called experiments of testing and experiments of application:

You are confronted with a problem in physics to be solved practically; in order to produce a certain effect you wish to make use of knowledge acquired by physicists; you wish to light an incandescent bulb; accepted theories indicate to you the means you have to secure certain information; you ought, I suppose, to determine the electromotive force of the battery of generators at your disposal; you measure this electromotive force: that is what I call an experiment of application. This experiment does not aim at discovering whether accepted theories are accurate or not; it merely intends to draw on these theories. In order to carry it out, you make use of instruments that these same theories legitimise; there is nothing to shock logic in this procedure. (Duhem 1954, p. 184)

In To Save the Phenomena, Duhem assimilated the notion of application to the notion of empirical adequacy: in the model provided by astronomy, the domain of application of theory (such as for instance, Ptolemy's) coincides with its domain of empirical adequacy. (If Ptolemy's theory can be applied to all kinds of observed motions in the sky, then it is an empirically adequate theory of celestial motion.) In other words, the cases that confirm the theory are precisely those to which the theory gets applied. However this view, which construes a theory's applications as strict deductions from theory, is 
not a consequence of Duhem's empiricist epistemology. It is instead a consequence of taking astronomy to provide the model for the application of theories. Astronomy provides a handy picture, one in which application and confirmation go hand in hand. In this picture the domain of application of a theory always coincides with its domain of empirical adequacy. But there is no reason why physics as whole ought to accord to this picture, and there is no reason why empiricism ought to be committed to it.

\section{The Semantic Conception of Scientific Theories}

The semantic conception of scientific theories has antecedents in work by Beth and Von Neumann from the 1930's through to the 1950's. Patrick Suppes developed it into a comprehensive account of scientific theories in a series of papers in the late 1950 's. ${ }^{3}$ Van Fraassen expresses the basic tenet of the semantic view as follows:

To present a theory, we define the class of its models directly, without paying any attention to questions of axiomatizability, in any special language, however relevant or simple or logically interesting that might be. And if the theory as such is to be identified with anything at all -if theories are to be reified - then a theory should be identified with its class of models. (Van Fraassen 1989, p. 222) ${ }^{4}$

There are however, many kinds of models. What sort of models is the semantic conception appealing to? Defenders of the semantic conception seemingly differ in their answers to this question. I will begin by distinguishing two kinds of models. I call the first kind semantic models, for lack of a better term. These models are also sometimes referred to as interpretations, although this terminology is somewhat misleading: a semantic model is not only an interpretation, but an interpretation and a structure. The structure is composed of a domain $D$ of objects and some relations $R_{i}$ defined over the elements in that domain, and can be denoted as $S=<D, R_{i}>$. A firstorder language $L$ is specified which contains logical constants, names and n-place predicates. An interpretation of the language $L$ in terms of the structure $S$ is a mapping from the constants in a language into the elements of the domain $D$, and from the predicates in the language into the relations $R_{i}$ in the structure. In order to identify a

\footnotetext{
${ }^{3}$ See Suppes 1961, 1969.

${ }^{4}$ By "defining directly", van Fraassen here means "not indirectly in terms of the satisfaction of axioms in a particular language".
} 
particular interpretation we need to specify both the structure $S$ that constitutes the mapping's range, and also the language $L$, including the syntax, that constitutes its domain. Thus, the definition of a semantic model is always tied to a particular language.

The second kind of models I call simply structures. To specify these we need not specify a particular syntax; structures are not necessarily tied to any first-order language. These are typically mathematical structures, defined over a domain of mathematical objects. In van Fraassen's version of the semantic conception a scientific theory is a collection of models of this kind:

The impact of Suppes' innovation is lost if models are defined, as in many standard logic texts, to be partially linguistic entities, each yoked to a particular syntax [...]. Models are mathematical structures, called models of a given theory only by virtue of belonging to the class defined to be models of that theory. (Van Fraassen 1989, p. 366)

This suggests that the name of the "semantic" conception of scientific theories is perhaps misleading. For this name brings to mind the syntax/semantics distinction in linguistics, and the model-theoretic notion of an interpretation of a particular language; while, as a matter of fact, the "semantic" conception defends the view that scientific theories are families of mathematical structures - and these are not to be thought of as providing an interpretation of any particular language. This "structuralist" construal of the semantic conception has an important advantage over the "semantic-model" construal. It provides an answer to the charge, made by Michael Friedman and John Worrall, that there is no essential superiority of the semantic over the syntactic conception. ${ }^{5}$ If the models that the semantic view refers to were models of the first kind, i.e. semantic models, the semantic and syntactic characterisations of a theory would be provingly equivalent. Given an elementary class of models (call it $N$ ), we know by the completeness theorem of first order logic that there must be a set of axioms $A x$ in the language of first order logic, which is satisfied by this and only this set of models. So talk about models (semantic models) is equivalent to talk about the axioms (in the language of first order logic). As Michael Friedman puts it:

Let us follow van Fraassen in identifying a theory with a class of models or structures. Suppose, however, that the class of models in question is

${ }^{5}$ Friedman 1982; Worrall 1984. 
a so-called "elementary class": i.e. that it contains precisely the models of some first-order theory $\mathrm{T}[\ldots]$. Then the completeness theorem immediately yields the equivalence of van Fraassen's account and the traditional syntactic account. (Friedman 1982, pp. 276-277)

Van Fraassen has answered this criticism in two stages. First he has argued, following Patrick Suppes, that first-order logic will normally be insufficient for the formalisation of any interesting theory. The Friedman-Worrall equivalence claim goes through only if a set of axioms in the language of first order logic exists of which the family of models is an equivalence class. The completeness theorem then guarantees the equivalence claim. But, any physical theory that appeals to the real number continuum cannot be formally axiomatised in the language of first order logic. And virtually no interesting physical theory can make do without employing the real numbers and differential calculus.

The completeness theorem is, as Patrick Suppes has emphasised, ${ }^{6}$ irrelevant in the formalisation of any interesting physical theories -in his famous slogan: "philosophy of science should use mathematics and not meta-mathematics". ${ }^{7}$ Imagine that a scientific theory is presented in just the way that the semantic view advocates, i.e. by specifying a class of structures directly, without appealing to any particular language. Call this set of structures $T$. Suppose further, that in specifying these structures we need to mention the real number continuum. We can try to formalise $T$ in some particular natural or artificial language $L$. We will write a set of axioms $A x$ which we can correlate with the class of semantic models in $L$ that make the axioms in $A x$, and only those axioms, true. This is an equivalence class of models in $L$ : call this set of semantic models $N$. However, the real number continuum is infinite, and the Löwenheim-Skolem theorem guarantees that there will be many models in $N$ not isomorphic to any of the structures in $T$. So $N$ is not a very good representation of $T$; and $A x$ is a rather poor axiomatisation of $T$ in the language $L$ (note however, that no better axiomatisation of $T$ in $L$ is available, as the same problem recurs with any other set of axioms).

This suggests, however, a more general thought: the class of models $M$ that constitute a scientific theory is simply not an elementary class - that is, there is no set of axioms (in any language) that is satisfied by all the models in the class and by only those models.

${ }^{6}$ Suppes 1970, chapters 1, 2.

${ }^{7}$ Quoted by van Fraassen 1980, p. 65. 
Indeed van Fraassen's second defence has consisted in stressing the distinctions between what I call "semantic models" and structures. He has written: "if a theory is to be identified with the set of its models, is that set an elementary class or not? This question makes sense only if we construe 'models' as referring to the models of some particular language" (Van Fraassen 1985, p. 302).

And, more recently:

In a trivial sense, everything is axiomatizable, because a thing must be described in order to be discussed at all. But for logicians, "axiomatisable" is not a vacuous term, and a scientific theory need not be axiomatizable in their sense - or as they say, the family of models may not be an elementary class. (Van Fraassen 1989, p. 211)

Consider again the case of $T$, defined directly by delimiting its set of structures. $T$ is not a set of semantic models, i.e. it is not a set of models in a particular language. The set $N$ is a set of semantic models: it is "a sort of image of $T$ produced through the lens (which may be more or less distorting) of the specific chosen language" (Van Fraassen 1985, p. 302). But moreover, as we saw, $N$ is typically too large - it contains plenty of structures not isomorphic to any structures in $T$. Some unspecified subset of $N$, call it $T^{*}$, will contain precisely those structures that are isomorphic to those in $T$, accompanied by interpretations of the syntax of $L$. So $T^{*}$ is a more perfect image of $T$ in terms of models of $L$ than is $N$. But if $T^{*}$ is some unspecified subset of $N$, there is no guarantee that it will turn out to be an elementary class. In fact, it is now clear that the same set of structures can form an elementary class of semantic models in a language $L^{\prime}$, given some interpretation of the syntax of $L^{\prime}$, while not forming an elementary class of semantic models in a different language $L$, however interpreted. The theory $T$ is, unlike $T^{*}$, not a set of semantic models that by accident fail to form an elementary class. For the elements of $T$, which constitute the theory, are not semantic models; they are instead a set of what I have called structures.

When a theory is presented, according to van Fraassen's semantic conception, it is not a set of semantic models that is given; rather it is a set of structures. It is then possible to circumvent the FriedmanWorrall equivalence claim - since structures on their own do not have the property to form "elementary classes". To define an elementary class of models we need a structure and an interpretation of the 
structure in a particular language. Semantic models implicitly allow us to do that, since they themselves specify a language; but a bare structure does not specify any language. It simply makes no sense to ask of a set of structures whether they constitute an elementary class.

\section{Empirical Adequacy within the Semantic Conception}

In this section I present two alternative accounts of empirical adequacy within the semantic conception of scientific theories, in terms of "embedding" and "reduction" respectively.

\section{1. Van Fraassen's Embedding}

Van Fraassen has characterised empirical adequacy as an embedding relation between two different structures:

To present a theory is to specify a family of structures, its models; and secondly, to specify certain parts of those models (the empirical substructures) as candidates for the direct representation of observable phenomena. The structures which can be described in experimental and measurement reports we can call appearances; the theory is empirically adequate if it has some model such that all appearances are isomorphic to empirical substructures of that model. (Van Fraassen 1980, p. 64)

In van Fraassen's view theories and phenomena alike are represented by sets of points in phase space, or alternatively by settheoretical structures. For convenience I'll use the latter formulation. A set-theoretical structure $\left.<D, R_{i}\right\rangle$ consists of a domain $D$ of some cardinality, and a class of relations $\left\{R_{i}\right\}$ between n-tuples of objects in the domain. Theories save phenomena by embedding phenomenological structures within theoretical structures. The technical notion of embedding is a follows. A phenomenological structure $P=<A$, $P_{j}>$ can be embedded into a theoretical structure $T=<B, T_{i}>$ (with $j \leq i$ ), if the former is isomorphic to an "empirical substructure" $E=<C, T_{j}^{\prime}>$ of the latter. The domain of the substructure is strictly a subset of the domain of the theoretical structure $(C \subseteq B)$. The relations $T_{j}^{\prime}$ that appear in the empirical substructure are the restrictions of the relations $T_{i}$ to the smaller domain of objects that appear in the empirical substructure $\left(\left\{T_{j}^{\prime}\right\} \subseteq\left\{\left.T_{i}\right|_{C}\right\}\right) .^{8}$

${ }^{8}$ See e.g. Suppes 2002, p. 62 . Relations over a domain can be defined extensionally by describing sets of n-tuples of objects in the domain. Thus a one-place relation 
It is important to stress that there are not just two structures, but three: the theoretical structure, the phenomenological structure, and the appropriate "empirical" substructure of the theoretical structure (see figure 1). The empirical substructure contains only relations that are already in the theoretical structure $\left(\left\{T_{j}\right\} \subseteq\left\{T_{i}\right\}\right)$, restricted to the new domain $\left(T_{j}^{\prime}=\left.T_{j}\right|_{C}\right)$. The theory would fail to embed the phenomenological structure if there were new relations defined on the domain of the phenomenological structure that do not appear in the theory. This is a sensible requirement: the theory could never account for all the richness and complexity of the phenomena if the phenomena were structured in ways the theory could not capture.

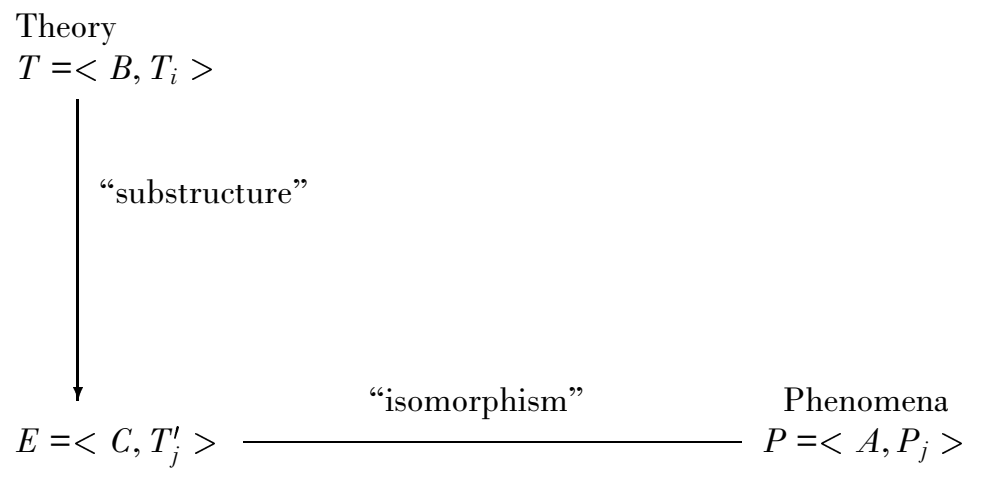

FigURE 1. Van Fraassen's embedding

Hence this characterisation of empirical adequacy amounts to the following: a theory is empirically adequate if and only if for every phenomenological structure in the theory's domain, the theory contains some structure that possesses an empirical substructure isomorphic to that phenomenological structure. ${ }^{9}$ If it is to be empirically

can be defined simply as a subset of the domain, a two-place relation as a set of pairs of objects chosen from the domain, and more generally an n-place relation can be defined by presenting a set of n-tuples of objects chosen from the domain. Hence a restriction to a smaller domain of a relation $T^{n}$, defined by a set $S$ of n-tuples, consists in taking out of $S$ all those n-tuples that contain at least one object which is absent in the smaller domain. The restricted relation $T^{\prime n}$ is then defined as the set of remaining n-tuples.

${ }^{9} \mathrm{I}$ am assuming that embedding is a necessary and sufficient condition for empirical adequacy. In the passage quoted earlier on, van Fraassen appears to present embedding as a sufficient condition only. However, the only if part is, I think, elliptical. Embedding is commonly understood to be necessary as well as sufficient and van Fraassen himself understands it as a necessary condition, for 
adequate the theory must put forward an object to stand for each object in the domain of the phenomena, and a candidate relation for every relation that appears in the phenomenological structure. (In addition, a theory may postulate "surplus" structure of course; a good scientific theory will typically do so.)

But the requirement of isomorphism between phenomenological structures and "empirical" substructures has further, important, consequences. An isomorphism is a one-to-one mapping, a function that maps every element in $C$ uniquely onto some element in $A$, and every relation in $\left\{T_{j}\right\}$ uniquely onto some relation in $\left\{P_{j}\right\}$. Formally, an isomorphism between two structures $P=<A$, $P_{i}>$ and $E=<C, T_{j}^{\prime}>$ is a function $f: A \rightarrow C$, such that if $x_{1}, x_{2}, \ldots, x_{n}$ form an n-tuple of objects in $A$ and if $y_{1}, y_{2}, \ldots$, $y_{n}$ form an n-tuple of objects in $C$, then: there is some n-relation $P_{i}$ which holds of $x_{1}, x_{2}, \ldots, x_{n}$ if and only if there is some n-relation $T_{j}^{\prime}$ which holds of $f\left(x_{1}\right), f\left(x_{2}\right), \ldots, f\left(x_{n}\right)$; and there is some $T_{j}^{\prime}$ which holds of $y_{1}, y_{2}, \ldots, y_{n}$ if and only if there is some $P_{i}$ which holds of $f^{-1}\left(y_{1}\right), f^{-1}\left(y_{2}\right), \ldots, f^{-1}\left(y_{n}\right)$. More concisely, an isomorphism between two structures $P=\left\langle A, P_{i}\right\rangle$ and $E=<C, T_{j}^{\prime}>$ is a function $f: A \rightarrow C$ such that $P_{i}\left(x_{1}, x_{2}, \ldots\right.$, $\left.x_{n}\right)$ iff $T_{j}^{\prime}\left[f\left(x_{1}\right), f\left(x_{2}\right), \ldots, f\left(x_{n}\right)\right]$, for any $x_{1}, x_{2}, \ldots, x_{n} \in A$; and $T_{j}^{\prime}\left[y_{1}, y_{2}, \ldots, y_{n}\right]$ iff $P_{i}\left[f^{-1}\left(y_{1}\right), f^{-1}\left(y_{2}\right), \ldots, f^{-1}\left(y_{n}\right)\right]$, for any $y_{1}$, $y_{2}, \ldots, y_{n} \in C$.

It follows that the domain $C(B \supseteq C)$ that the theory puts forward as a candidate for representing the phenomena must be of the same cardinality as the domain of objects $\mathrm{A}$ in the phenomenological structure. The number of relations $T_{j}$ defined over this domain must be identical to the number of relations $P_{j}$ that appear in the phenomenological structure. And, although the objects and their properties (represented by the n-place relations $P_{j}$ on $A$ ) need not be preserved with the mapping, the properties of the relations that denote those properties must be so preserved. ${ }^{10}$ The existence of an

instance, in his arguments against causal explanation of the EPR-Bohm correlations; — see van Fraassen 1982, and Suárez 2004, for a critical review.

${ }^{10}$ A word is due on my usage of the terms "property" and "relation". I draw no ontological distinction between the two: properties might be relational, and oneplace relations are monadic. I reserve the word "relation" for the properties of the elements of a structure that is intended to represent something else; and the word "property" for the properties of the objects so represented. Hence when I speak about the phenomenological structure $P$ in its relation to the phenomena that it represents, I talk about its $\left\{P_{i}\right\}$ as "relations". But when I speak about $P$ in its 
isomorphism between two structures is, so to speak, a statement that the structures have identical properties: isomorphism is structural identity. ${ }^{11}$

There is one fundamental difference between this characterisation of empirical adequacy in the semantic view, and the old syntactic criterion of empirical adequacy. ${ }^{12}$ The old criterion turns out to be empty - because it is not possible to isolate the empirical content of a theory merely by considering only those sentences that can be formed out of the observational vocabulary, since some of those sentences might entail facts about the unobservable world. ${ }^{13}$ By contrast, the semantic view allows us to unambiguously isolate the empirical import of a theory - as simply the set of substructures the theory puts forward as candidates for the representation of phenomena. And there is no reason why we should have to refer to these objects and relations, when we speak about them, in a purely "observational" language. Such requirement would only follow if the models that, on the semantic view of theories, constitute a scientific theory were semantic models. For in that case, as Michael Friedman makes clear:

The empirical substructures in question are definable in the language of [the theory ... ]. Consider one of van Fraassen's empirical substructures: a set of objects (the observable objects) together with appropriate properties and relations. Now look at the terms the theory uses to denote these properties and relations: these comprise our observational vocabulary. Look at the sentences built up from these terms by truth functions and quantifiers restricted to the set of observable

relation to the theoretical structure $T$ intended to represent $P$ then $I$ refer to the $\left\{P_{i}\right\}$ as "properties".

${ }^{11}$ The relation $T_{j}^{\prime}$ put forward by the theory to match up the relation $P_{j}$ in the phenomenological structure will not hold of the same objects because the phenomenological structure is not part of the theoretical structure. As a relation can always be defined extensionally by appeal to sets of n-tuples of objects in its domain, and as the domains of the phenomenological and the theoretical structure will typically be different, it follows that the relations themselves will be different. In other words, isomorphism between structures neither requires that the objects in the structure be the same, nor that the properties of those objects be identical. However, if there is to be an isomorphism between the structures the domains $A$ and $C$ must be of equal cardinality, and the corresponding relations $T_{j}^{\prime}$ and $P_{j}$ must possess identical properties. For instance, an equivalence relation over a domain of cardinality $x$ is mapped onto another equivalence relation over a domain of equal cardinality, etc.

${ }^{12}$ For an account of the relation between theory and phenomena in the old syntactic conception of scientific theories, see Nagel 1979, chapter 5.

${ }^{13}$ Suppe 1974, pp. 80-86. See also van Fraassen 1980, p. 54. 
objects: these will be just the observational sentences. $T$ will be empirically adequate in van Fraassen's sense just in case its (syntactic) consequences within this class of observational consequences are true. (Friedman 1982, pp. 276-277)

If the class of models that constitutes the theory was an "elementary class" there would be a syntactic representation of the theory $T$, and also a syntactic representation of the empirical content of this theory. In rejecting the construal of models as semantic models, we have pre-empted this possibility: a theory is a collection of models, but, fortunately, these are not semantic models; hence they cannot be said to form an elementary class. So there need be no corresponding syntactic characterisation of any of the features of a scientific theory, including its empirical adequacy.

\subsection{Friedman's Model-Submodel Reduction}

Embedding allows nicely for an empiricist intuition about confirmation: the degree of confirmation of a theory does not necessarily measure the likelihood that the theory is true but, rather, the likelihood that the theory is empirically adequate. According to the embedding conception a theory provides representations of the phenomena by advancing mathematical structures and delineating those substructures that are intended to directly model the phenomena. The mathematical structures are constituted by certain entities (such as numbers and n-tuples of numbers, lines, planes, manifolds, vectors and n-tuples of vectors, etc., etc.) and some relations defined over them (operations such as norm, angle, scalar product, trace, or, for instance, the relations of space-like, light-like and time-like separation in relativity theory). Of course, theories may be more or less successful in this task of representing, and a theory's accumulated degree of confirmation measures just its overall success in representing phenomena. For convenience, I will refer to this picture of the relation between theories and phenomena as the "representational" picture, or "representationalism" for short.

Notice that representationalism allows van Fraassen's constructive empiricism but does not require it. Someone who believes that science must account for more than observable phenomena can perfectly well adopt the representational picture. For instance someone who thinks that the phenomenological structures can also represent nonobservable entities and properties would be committed to the thought that the empirical content of a theory goes beyond its observational 
predictions. This thought, however, can easily be accommodated within the representational picture by expanding the corresponding "empirical" substructures so that they too contain a representation of those non-observable entities. It would even be possible to expand the set of "empirical" substructures so that they cover the whole theoretical structure, thus demanding that our theories represent the whole world. The constructive empiricist thus makes an additional commitment - additional that is, to the bare representational pictureby restricting the phenomenological structures to observable entities and their properties, and advising us to remain agnostic about the existence of the unobservable entities and processes postulated by scientific theories. According to this additional commitment, we may choose to accept a scientific theory as a research programme and we may have confidence in its future predictions, but the only belief that is involved in such acceptance is the belief that the theory is empirically adequate, not the belief that it is true. In van Fraassen's well known phrase: "Science aims to give us theories which are empirically adequate; and acceptance of a theory involves as belief only that it is empirically adequate" (1980, p. 12).

Van Fraassen's selective epistemology implies that it is always possible to identify the empirical import of a theory, and to separate it from the "surplus" theoretical commitment. The semantic view, in its representational picture, provides a schema: those empirical substructures that the theory puts forward as models of the phenomena constitute the empirical content of a theory. ${ }^{14}$ We are then advised to believe only in the existence of those entities and processes that appear in the phenomenological structure (i.e. according to the constructive empiricist, those entities that are directly observable with the unaided senses); and we are told that we are not required to believe in the existence of the unobservable entities that the theory postulates.

${ }^{14}$ This is the source of a pervasive misunderstanding of van Fraassen's epistemology. For instance, in an interesting recent paper William Demopoulos argues that constructive empiricism falls prey to Newman's objection to Russell's structuralism, and is thus incoherent. Demopoulos' argument is involved and I will not here try to assess it; but it is worth pointing out that he frames constructive empiricism mistakenly in the reduction picture, and not in the representational picture: "to accept a theory as empirically adequate [according to constructive empiricism] is to hold that 'the phenomena' form a substructure of a model belonging to the class of models that is the theory", and "to believe a theory true is to hold that it contains the world among its objects" (Demopoulos 2003, p. 389). This is not the way that van Fraassen would frame the issue. 
Michael Friedman, on behalf of the scientific realist, has put forward an argument against the representational picture. In his view, a more appropriate account of the relation between theory and phenomena (particularly in the context of space-time theories) is rendered by the notion of model-submodel reduction. In contrast to an embedding, a reduction requires only two structures: the theoretical and the phenomenological structure. A theory is empirically adequate if it can literally subsume the phenomena (see figure 2 ). In a reduction the domain of the phenomenological structure is a proper subset of the domain of the theoretical structure $(A=C \subseteq B)$, and the properties found in the phenomena are exactly the same properties that the theory postulates its mathematical entities possess $\left(\left\{P_{i}\right\}=\right.$ $\left.\left\{T_{j}^{\prime}\right\}=\left\{\left.T_{j}\right|_{C}\right\}\right)$. As Friedman writes: "Under this construal $T$ functions as a genuine explanation or reduction of the properties of $P$, for elements of $P$ are literally identified with elements of $T$ " (1983, p. 236).

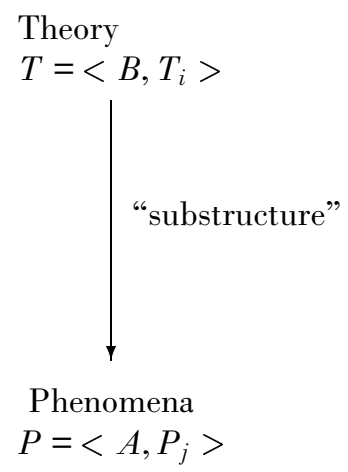

FigURE 2. Friedman's "reduction"

As a consequence, on this alternative "reduction" picture, if a theory is shown to account for some new phenomenon, this constitutes a reason to believe that the whole theory is true -including the surplus theoretical relations $\left\{T_{j+w}\right\}$ (with $0 \leq w \leq i-j$ ), and the "surplus" domain $B-C$. For it would make no sense to claim, on this view, that the phenomenological structure is true but the theoretical structure is not, when the phenomenological structure is actually a part (a "chunk") of the theoretical structure. Therefore, in this reduction picture, degree of confirmation is a measure of the likelihood that the theory is true. 
The main argument in favour of constructive empiricism (and its close ally in the philosophy of space and time, relationalism) is that a scientific theory has exactly the same empirical consequences on the representational picture as on the reduction picture. Therefore, a theory has exactly the same degree of confirmation on either picture. As the representational picture is committed to the existence of fewer entities, refraining as it does from reifying any theoretical entities, it is always preferable on grounds of ontological parsimony.

An initial objection to the representational picture is that it is not possible to do without theoretical "surplus" in the description of the phenomena. This "surplus" will often induce theoretical properties and relations on objects in the phenomenological domain - which are normally required to state accurate laws about the behaviour of these objects. But, the objection continues, the representational picture implies that no "surplus" can ever be employed in the description of the phenomena. Asserting that the phenomenon $P=\left\langle A, P_{j}^{n}\right\rangle$ is merely embeddable in a theory $T=\left\langle B, T_{i}^{n}\right\rangle$ will not induce the necessary theoretical properties and relations. If $T_{v}^{l}(i>v>j)$ is one such theoretical relation then, unless $T_{v}^{l}$ is definable from the set of phenomenological properties $\left\{T_{j}^{\prime n}\right\}$, there will be automorphisms of the domain of the empirical substructure $C$ that will leave the isomorphism with the phenomenological structure entirely intact while crucially altering the extension of $T_{v}^{1}$ in the empirical substructure. ${ }^{15}$ In other words, there will be two embeddings $f$ and $g$ of $P$ into $T$ that take every $P_{j}^{n}$ into its corresponding $T_{j}^{\prime n}$ and yet such that, for some object $c \in C$ it will be the case that $T_{v}^{\mathrm{l}}(f(c))$ and $\neg T_{v}^{\mathrm{l}}(g(c))$.

This objection to the representational picture is, however, not decisive. For there is no reason why the representationalist cannot simply "expand" the empirical substructure relative to the theoretical structure by restricting this new relation $T_{v}^{l}$ to the domain $C$ of the empirical substructure. That is, when the correlate of some theoretical relation is found to apply to some phenomenon, the representationalist will insist that such relation be brought into the empirical substructures of the theory. Suppose, to use a well known modelling example, that it was found that gas molecules, like billiard balls, are actually coloured; the representationalist will ipso facto want to introduce the relevant relations into the appropriate substructure. The representationalist might even be justified to introduce all "surplus"

${ }^{15}$ See Friedman 1983, p. 240. 
theoretical relations $\left\{T_{j+w}^{n}\right\}$ (with $w \leq i-j$ ), appropriately restricted to $C$, into the "empirical substructure". ${ }^{16}$

Friedman, who is well aware that a different strategy is needed to defend the reduction picture, has proposed to block the main premise of the above argument in favour of the representational picture. He denies that in all occasions a scientific theory will gain the same amount of confirmation on the representational picture as on the reduction picture. In particular, he claims, in cases of theory-unification there is a substantial boost in confirmation for the individual theories that make up the unified theory whenever the unified theory comes up with a successful new prediction in a different domain. The reduction picture can accommodate this fact naturally, while the representational picture is unable to do so; thus providing an argument against representationalism generally.

Morrison (1990) has given an interesting reply to Friedman. However, this debate, which is fascinating in its own right, is not directly relevant to the concerns of this paper. The reductionist and the representationalist can equally account for the theoretical induction of properties and relations into the phenomena; they agree on the degree to which a particular phenomenon confirms a particular theory; and, more importantly, they agree that a theory always obtains confirmation from phenomena that it accounts for (although, naturally, they might disagree as to whether increased confirmation indicates that the theory is more likely to be true, or just more likely to be empirically adequate).

Moreover, they agree on what the logical relation is between their own positions. The representationalist thinks that there may be many

\footnotetext{
${ }^{16}$ Admittedly, however, the above argument may cut some way against the specific representationalism advocated by the constructive empiricist. For the introduction of $T_{v}^{l}$ into the "empirical" substructure entails the existence of a corresponding property in the phenomenological structure, under the assumption that both structures continue to be isomorphic. Is the constructive empiricist happy to introduce theoretical relations among the appearances, if it is in the restricted form in which they are taken to apply to observable entities only? I am not sure. Perhaps van Fraassen's internalism can help here. For internalism - the thesis that it is up to science itself to decide what to count as observable and what not- enables us to bring into phenomenological structures relations that were previously thought to be unobservable. (N.B.: internalism is built into van Fraassen's definition of a scientific theory: "to present a theory is to specify a family of structures, its models; and secondly, to specify certain parts of those models (the empirical substructures) as candidates for the direct representation of observable phenomena" (van Fraassen 1980 , p. 64; my emphasis). In any case, representationalism in general is in no trouble at all here - since it is not committed to the view that the entities and relations in the phenomena should be observable.
} 
different ways of mapping a phenomenological structure onto empirical substructures of a theory (in other words there may be different mappings: $f$ and $g$ above examples), while the reductionist thinks that there is only one possible mapping, namely identity. Hence, they both agree that reduction is logically stronger than embedding. And this is important because it shows that if a theory is unable to embed a particular phenomenon then it cannot be empirically adequate on either characterisation of what it means for a theory to be empirically adequate. Such a theory, therefore, cannot draw any confirmation at all from that particular phenomenon.

\section{The Empirical Basis of Science}

I have been discussing different conceptions of scientific theories, with special emphasis on the relation between theory and phenomena. Van Fraassen and Friedman have established the requirements that this relation must fulfill if the theory is to receive confirmation, on a broadly empiricist and realist construal respectively. Their work is heavily informed by a conception of theories as structures. But, so far, I have had little to say about the other relata of the confirmatory relation, namely phenomena. A more detailed discussion of what constitutes phenomena is imperative, however, for van Fraassen's and Friedman's epistemological purposes. Theories are to receive confirmation from the phenomena they account for; but unless we have good grounds for believing that the phenomena are true, van Fraassen's and Friedman's strictures will count for nothing in persuading us to believe in the truth of our theories. In other words, phenomena must constitute a solid epistemic basis for scientific theories.

Both van Fraassen and Friedman assume that it is possible to describe a phenomenon as a structure. This is of course essential for their proposals to work, as both embedding and reduction are defined as relations between structures. Van Fraassen mentions that he first saw this kind of formalised structures of the phenomena in the works of Wojcicki, Przelecki, Dalla Chiara and Toraldo di Francia and, particularly, Suppes' classic paper "Models of Data". ${ }^{17}$

\subsection{Models of Data}

Suppes argues that there is a hierarchy of set-theoretic models all the way down from theory to experimental data. He concentrates

${ }^{17}$ Wojcicki 1974; Przelecki 1969; Dalla Chiara and Toraldo di Francia 1979; Suppes 1969. 
on statistical theories, where the basic hierarchy contains five layers: models of theory, models of experiment, models of data, methods and models of experimental design, and ceteris paribus conditions on extraneous factors in the experiments actually performed. I will not review Suppes' paper in detail here. ${ }^{18}$ It is enough to emphasise that according to Suppes, the relation between theory and data is very complex: the theory contains continuous distributions but the data is finite, and one has to figure how well the relative frequencies in the finite sample match the distribution function. The theory has to be "prepared" to meet the demands of the experiment, by truncating the continuous distribution $P$ over the infinite set $X$ into a discrete measure over the finite set. The data has to be similarly prepared in a form that the theory is able to account for, by selecting those runs whose frequencies of reinforcements fit at least approximately the conditional probabilities in the model of the experiment.

In practice the decisions to "prepare" the theory and the data for each other - by imposing certain constraints on the probability distributions in the theory, or by selecting a subset of experimental runs - can only be made on a case-by-case basis. There are no general rules. These judgements demand practical skills and a fine sense for the details of the experiment; they demand a considerable amount of tacit knowledge and a careful consideration of the particular conditions under which the experiment is performed. Here I have not dealt with the sort of detailed considerations that play a role in Suppes' example. (They are many and subtle; Suppes himself refrains from discussing them all in detail.) The main point that I want to emphasise, following Suppes, is that theories do not confront raw data, but rather, specifically tailored, contrived and highly conceptualised "data models".

\subsection{The Empirical Basis: Data or Phenomena?}

The same point has been reinforced and expanded in an interesting way by Jim Bogen and Jim Woodward, who draw the distinction between data and phenomena. Facts about data describe sets of datapoints on a graph, records of scintillation spots on a fluorescent screen, registered tracks in a bubble chamber, etc. Data live in the laboratory, they are highly dependent upon the particular experimental context in which they are observed and recorded; and they are generally short-lived: as better, more accurate experiments are

${ }^{18} \mathrm{It}$ is discussed and reviewed in detail in my PhD thesis: Suárez 1997. A comprehensive discussion and elaboration can be found in Mayo 1996, chapter 6 . 
performed, the data from previous experiments tend to get ignored or reinterpreted. By contrast, phenomenological facts describe wellestablished general patterns of nature, and effects. For instance, the fact that metals dilate in the presence of heat is a rather typical phenomenological fact. Phenomena constitute firmly established bodies of scientific knowledge; they are independent of the particular conditions under which experiments are performed; and, besides, they are relatively theory-independent, in the sense that they tend to "last long": facts about phenomena are manifestly impervious to theorychange.

Bogen and Woodward's main claim is that phenomena, not data, constitute the empirical basis of science. First, they argue that scientific theories are advanced by scientists as explanations of phenomena, but are not normally intended as explanations of data: "typically, scientific theories are expected to provide systematic explanations of facts about phenomena rather than facts about data" (Bogen and Woodward 1988, p. 322). Theories must account for (i.e. be empirically adequate to) phenomena, not data. Secondly, Bogen and Woodward argue, what is observed and gets recorded during an experiment is data, not phenomena. Phenomena are instead inferred from bodies of data: "data, which play the role of evidence for the existence of phenomena, for the most part can be straightforwardly observed. By contrast [...] phenomena are detected through the use of data, but in most cases are not observable in any interesting sense of that term" (Bogen and Woodward 1988, p. 306).

Bogen and Woodward's account of phenomena has strong similarities with Suppes' account of data-models, but there is one important difference. For Suppes data-models are primarily employed to test scientific theories. This is indeed the reason why the data-model must be prepared in the light of the theory's assumptions and requirements - so the data can be put to use as a meaningful test of the theory. By contrast, models of the phenomena are constructed to describe stable phenomena or to elucidate them, not to test theory; although they may be constructed with the help of theory. One example is Fritz London and Heinz London's early 1933 model of superconductivity. ${ }^{19}$ The Londons' aim was not to test electromagnetic theory, but to refine our understanding of superconductivity phenomena. Their model constituted a model of the phenomenon of superconductivity since it was able to predict both resistanceless conductivity and the

${ }^{19}$ See Cartwright, Shomar and Suárez 1995; Suárez 1997, 1999; French and Ladyman 1997. 
Meissner effect. The claim that superconductors expel magnetic flux is surely a phenomenological claim, if any claim ever is: it is quite impervious to theory change, and its truth does not depend upon any of the conditions in the concrete experiments performed to verify it. In contrast, a data-model, which may record the average experimental rates of expulsion of flux in the Meissner-Ochsenfeld experiment, is a highly contextual model; it may be true of the data collected in that particular experiment, but it cannot constitute a model of the Meissner effect per se.

\subsection{Hierarchies of Models}

According to Bogen and Woodward a phenomenon is not simply an inductive generalisation of data, although it may be inferred from the data. What exactly is the evidential relationship between data and phenomena? Mathias Kaiser (1991) has proposed an account in terms of hierarchies of structures. In his picture, a model of a phenomenon $S_{k}$ sits at the top of a hierarchy of models of data (see figure 3 ).

$$
\begin{aligned}
& S_{k}=<D_{k}, n_{k}^{1}, \ldots, n_{k}^{m}> \\
& S_{k-1}=<D_{k-1}, n_{k-1}^{1}, \ldots, n_{k-1}^{m}> \\
& \ldots \\
& \\
& \ldots \\
& S_{1}=<D_{1}, n_{1}^{1}, \ldots, n_{1}^{m}> \\
& S_{0}=<D_{0}, n_{0}>
\end{aligned}
$$

FigURE 3. Layers of Structures

According to Kaiser, the bottom layer's structure has as its domain only objects that can be directly observed, and the relation $n_{O}$ is ostension (i.e. the only property ascribed to the objects at this level of description is that of "being picked out by ostension"). ${ }^{20}$ The next structure up is defined by introducing a set of new relations

${ }^{20}$ In another puzzling paragraph Kaiser asserts that the objects in the bottom layer are "all those things that are to be subjected to scientific scrutiny, i.e. that are to be measured, weighed, radiated, dissoluted, accelerated, etc." (1991, p. 125). It is not the case, however, that everything measurable is definable by ostension, or even 
$\left\{n_{i}\right\}$ over a perhaps different domain $\left\{D_{i}\right\}$. The whole hierarchy is defined in a number of steps, or layers, each of which corresponds to a change of elements in the domain, or a change in the set of relations. These operations are intended to represent scientific practice, hence they correspond to operations and redefinitions of the domain that are actually performed in the course of scientific research. The passage from one structure to the next one up is grounded upon what Kaiser calls "inference tickets" which come in two kinds: as warrant for a re-description of the domain, or as legitimating new types of operations and computations. Theoretical knowledge is involved - although not necessarily knowledge derived from one and the same theory. "Inference tickets" are provided by probing techniques and instruments, error theory, test-methods to eliminate useless samples, statistical techniques for the treatment of data, etc.

Kaiser's hierarchy of models of data has three interesting features. First, it represents a model of the phenomena as a structure $\left.S_{k}=<D_{k}, n_{k}^{i}\right\rangle$, as required by the advocates of the semantic view. Second, it allows for the fact that the construction of models of phenomena may be constrained (although not dictated by) theory, as in Suppes' example. Finally, it shows that an adequate scientific theory ought to account for the phenomena, which are represented by the top structure in the hierarchy of structures.

As a consequence, however, it is necessary to forgo the requirement that an empirically adequate theory must "embed" or "reduce" data: On Kaiser's proposal the model of the phenomena will normally fail to embed or reduce the lower models of the hierarchy. As in Suppes' example the changes in the domain and in the set of relations are not necessarily driven by one unique body of knowledge, so there is no guarantee that the domains and relations of structures in different layers will be appropriately "nested". Hence an empirically adequate theory (a theory that accounts for the phenomena) will typically not embed or reduce the data that serve as evidence for the phenomena. On Bogen and Woodward's view, however, this is as it should be: data rarely constitute evidence for a scientific theory;

observable. For example, many economic entities (inflation, unemployment, bullion) can be measured very accurately, yet can never be truly observed. In this respect physics is actually not that different; consider for instance measurements of electric currents in circuits by means of amperometers, or measurements of field strengths by induction techniques. I take it that Kaiser's conflation is a result of his narrow focus on his case studies in paleomagnetism. 
instead the evidence for or against theories is typically to be found in well-established phenomenological facts.

\section{The Application of Scientific Theories}

I am now finally in a position to frame precisely the question at the heart of this paper.

Ian Hacking ${ }^{21}$ denies that scientific models are generally "doubly" models, i.e. models of phenomena as well as models of theory. Some examples of models of phenomena include early models for continental drift phenomena, the early models of the atom (the Bohr atom), the billiard ball model in phenomenological thermodynamics, models for stellar structure, gravitational lensing models. How do these models relate to models of theory: models of the continental drift theory, the hydrogen atom in quantum theory, models of the kinetic theory, models in the quantum theory of radiation, and the Friedmann models in cosmology? According to the advocates of the semantic view if a theory is to be empirically adequate its models must embed, or reduce, all models of phenomena in the domain of the theory. Can this account of empirical adequacy be used to describe the application of scientific theories? Does the domain of application of a theory coincide with its domain of empirical adequacy?

\subsection{Revisiting the London account}

In section 4 it was noted that embedding is a logically weaker constraint than reduction. A theory might fail to reduce phenomena, and yet a part of the theory may be shown to be isomorphic (structurally identical) to the phenomena. In this sense, embedding is a broader constraint than the alternative realist account in terms of reduction. It is not, however, an empty constraint: some model of a phenomenon may not be embeddable in any theory. There is no means of embedding a model of phenomena which contains relations having no structural counterpart in physical theory. Some models of the phenomena cannot be embedded in any theory, not even in those theories employed to build the models in the first place. Some examples include gravitational lensing models in cosmology, stellar structure models in astrophysics and models of SQUIDS (superconducting quantum interference devices).

${ }^{21}$ See Hacking 1983, p. 216. 
The model that Fritz and Heinz London gave for superconductivity is another instance. Let me review the model from the point of view of the semantic conception of theories. In this case there are three structures. First, the theory of classical electromagnetism — which I take to include at least Maxwell's equations. Second, the "acceleration equation" model of Becker, Sauter and Heller, which entails the following relations for the magnetic flux inside the material:

$$
\Lambda c^{2} \nabla^{2}\left(\vec{H}-\vec{H}_{0}\right)=\vec{H}-\vec{H}_{0}
$$

where $H_{0}$ denotes the magnetic field at the time $t=0$. And, third, the London equation which models the Meissner effect by imposing a different constraint on the dynamics of the magnetic flux:

$$
\Lambda c^{2} \nabla^{2} \vec{H}=\vec{H}
$$

In previous works on this topic I provided two alternative pictures of the state of classical electromagnetic theory at the time of Meissner's discovery. ${ }^{22}$ First, I argued, it is possible to take "electromagnetic theory" in a historical sense, as constituted by all applications to electromagnetic phenomena known at the time. On this picture, the "acceleration equation" model was part of classical electromagnetic theory by 1933, and the following identification seems irresistible: the theory (classical electromagnetism) contains an empirical substructure (the "acceleration equation" model of Becker, Sauter and Heller), which it puts forward as a candidate for representing the phenomena (Meissner effect). As the Meissner effect is accurately described by the Londons' model, we can take the latter to provide its phenomenological structure. This might of course not be the only possible identification; but any model of the Meissner effect phenomenon must be isomorphic to the Londons' model (modulo some idealizations to do with penetration depths). Let me hence consider whether, with this identification in mind, classical electromagnetic theory can be said to account for the phenomena (in the sense of being empirically adequate).

We are looking for an isomorphism between the phenomenological structure and the empirical substructure. The London model is close

${ }^{22}$ Suárez 1999, and 1997, chapter 2. 
to being isomorphic to the "acceleration equation" model. First, the two domains are isomorphic: for every physical entity (i.e. $\mathbf{j}, \mathbf{H}$, etc.) in the domain over which the "acceleration equation" model is defined, there is a corresponding entity in the domain of the London model. Second, at least one relation over the domain is isomorphic, namely the relation that accounts for a constant current in the absence of external fields, expressed in the equation:

$$
\nabla \Lambda \frac{d \vec{j}}{d t}=-\frac{1}{c} \frac{d \vec{H}}{d t}
$$

This equation represents the structure that is in common between the London model and the "acceleration equation" model. Nevertheless, there fails to be complete isomorphism. The two models are not structurally identical in the required sense. Equation $(* *)$, which entails the "fundamental law" of superconductivity, yields a prediction that the "acceleration equation" model cannot match. In the London model we find a relation, lacking in the "acceleration equation" model, establishing that the magnetic flux after the phase transition is zero regardless of what the flux is before the transition. On the other hand, according to the "acceleration equation" model, there should typically be some non-vanishing flux after the transition. The phenomenological structure contains one relation - expressed in equation $(* *)$ - that is not in the theory, while the theory contains one relation - expressed in equation $(*)$ - that is not in the phenomena. The relation in the theory is an equivalence relation, characterised by an identity mapping that leaves the magnetic flux invariant, while the relation in the model is not even a symmetric relation (at least not when there is some initial magnetic flux).

There is a caveat, though: the fact that we have failed to find an empirical substructure of the theory isomorphic to the phenomena does not necessarily mean that the theory does not have one. We may have misidentified the correct empirical substructure of the theory: there could be another substructure, unknown to us, that is totally isomorphic to the London model. And yet, the "acceleration equation" model is inconsistent with the London model. So if the unknown substructure - the "good" one- is to account for the Meissner effect, it must contain at least one relation inconsistent with the "acceleration equation" model. This generates a paradox: 
the theory contains two substructures, intended for the same phenomenon, that yield mutually inconsistent predictions. A theory, if consistent, cannot yield inconsistent predictions for the very same physical phenomenon. Hence there must be at most one substructure of the theory that applies to superconductivity and, given the historical construal of electromagnetic theory, this must be the "acceleration equation" model — which fails to be isomorphic to the Meissner effect.

To sum up, on van Fraassen's characterisation of empirical adequacy as embedding - and, a fortiori, on Friedman's too-, classical electromagnetism, if construed in this historical sense, is not empirically adequate (of superconductivity phenomena). This cannot be very surprising - for we cannot possibly expect the "acceleration equation model" to be able to account for the Meissner effect, which it notoriously contradicts.

I hasten to add that this is not a conclusive result, since there is an alternative picture of electromagnetic theory, which takes classical electromagnetism to be constituted by nothing other than the deductive closure of Maxwell's equations. However, neither the acceleration equation nor the London models belong in this closure. So, on this abstract picture, the theory does not "reach far enough". To put it in Suppes' terms, the theory has not been adequately "prepared" to meet the phenomena: it can provide neither an accurate nor an inaccurate representation of superconductivity phenomena - it can provide no representation at all! The phenomenon of superconductivity simply lies outside the theory's domain of empirical adequacy, and the question of embedding, or reduction, of the Meissner effect simply does not arise.

\subsection{Partial Isomorphism}

Otávio Bueno has suggested that it may be possible to weaken the notion of isomorphism to a partial isomorphism, and then show that a partial isomorphism is possible between classical electromagnetism and the Meissner effect:

It seems the partial approach to embedding suggested above will fit Suárez's setting nicely. [...] Indeed the kind of new relation discovered at the phenomenological level might be represented by one of those partial relations that initially were not defined for some elements of the domain under investigation [...], and with the introduction of further bits of information, came to be defined. [...] Thus with the in- 
troduction of partial structures and partial isomorphism, the empiricist can answer Suárez's criticisms without difficulty. (Bueno 1997, p. 598)

However, Bueno's approach will not work in the historical picture of electromagnetic theory. A partial structure $S$ is a structure $<D, R_{i}>$, where some of the $R_{i}$ are partial relations (Da Costa and French 1990). A partial n-place relation $R$ over a domain $D$ is a triple $<R_{1}, R_{2}, R_{3}>$ where $R_{1}$ is the set of n-tuples that satisfy $R, R_{2}$ is the set of n-tuples that don't satisfy $R$, and $R_{3}$ is the set for which it is not known if they satisfy $R$. Given two partial structures $A=<D, R_{i 1}, R_{i 2}, R_{i 3}>$ and $B=<E, R_{i 1}^{\prime}, R_{i 2}^{\prime}, R_{i 3}^{\prime}>$, "the function $f: A \rightarrow C$ is a partial isomorphism if i) $f$ is bijective, and ii) for every $x$ and $y \in D, R_{i 1}(x, y)$ if and only if $R_{i 1}^{\prime}$ $(f(x), f(y))$ and $R_{i 2}^{\prime}(x, y)$ if and only if $R_{i 2}^{\prime}(f(x), f(y))$ " (Bueno 1997, p. 596).

Consider then an n-place partial relation $P_{1}=<P_{11}, R_{12}, R_{13}>$. No n-tuple of elements of $A$ can figure simultaneously in two of the sets defined by the extensions of $P_{11}, P_{12}, P_{13}$. A partial isomorphism between $P$ and $E$ is a bijection, and hence reduces to a set of three isomorphic mappings that take each of $P_{i 1}, P_{i 2}, P_{i 3}$ into the corresponding $T_{i 1}^{\prime}, T_{i 2}^{\prime}, T_{i 3}^{\prime}$. The problem that $\mathrm{I}$ am raising is that the London model contains a relation that is explicitly denied by the "acceleration equation" model and vice versa. Hence there is some n-tuple of objects over which some $R$ definitely holds in the London model and definitely doesn't in the "acceleration equation" model; and vice versa. This means that the relations that we are discussing are not in the "neutral analogy" part, $R_{i 3}$, of any of the partial relations. This must be true in both the London model, and the "acceleration equation" model. So there is no room to exploit the main resource of the partial isomorphism approach, namely that some n-tuples may neither belong to the extension of a relations, nor to the extension of its negation.

\subsection{Application versus Empirical Adequacy}

Suppose then that empirical adequacy is appropriately characterised by means of either reduction or embedding (or some weaker version of the latter). Neither the historical nor the abstract picture of classical electromagnetism will allow us to say the theory is empirically adequate of superconductivity phenomena. In one case it is actually inadequate, in the other case the question of empirical adequacy simply does not arise. But note that the failure of empirical 
adequacy does not seem to impugn the claim that both the acceleration equation model and the Londons' model were applications of classical electromagnetic theory to superconductivity. The Londons' model was moreover a rather successful application (to type-I superconductors at least): it accommodated both defining features of superconducting behaviour, and it had some predictive power: it yielded roughly accurate rates of expulsion of flux for the correct temperatures and values of the external magnetic field, and it accounted for the current and fields in superconducting spheres and wires appropriately.

At this point, embedding and reduction pull in one direction, theory application in another. Phenomena that the theory successfully gets applied to can be neither reduced to, nor embedded in, the theory. In other words, a theory's domain of application and its domain of empirical adequacy do not coincide. There are in principle two logical routes out of this dilemma. The first would be to relinquish the reduction or embedding conceptions, by weakening the concept of empirical adequacy. This would allow us to extend the concept of empirical adequacy to match that of application. Bueno attempts to follow this route, but he does not reach very far. This is not surprising: it would be counterintuitive and deeply problematic if the domain of empirical adequacy coincided with that of application, since there are bound to exist applications to phenomena that contradict the theory (as is the case with superconductivity and classical electromagnetic theory, at least when construed in the historical sense). And a theory that contradicts a phenomenon in its domain is by definition empirically inadequate.

The other route, which I prefer, accepts that a scientific theory can be applied to phenomena from which receives no confirmation boosts. The domain of application is generally much larger. We can then be as strict as we like with the domain of empirical adequacy and as liberal as we need to be with respect to application. And once this is accepted it is hard to see why we should have to weaken van Fraassen's embedding conception of empirical adequacy any further. The case study from superconductivity certainly does not provide a reason, since it does not per se invalidate the embedding approach to empirical adequacy. On the contrary, if the formal notion of embedding is to provide an extended characterisation of empirical adequacy, we should hope that it fails here, since we know that in this case the theory, as understood in the extended historical sense, is empirically inadequate. And indeed van Fraassen's embedding fails: the theoretical structure does not embed the phenomenological structure. This 
vindicates van Fraassen's notion of embedding against any weakening such as partial embedding; but it simultaneously undermines the identification of the theory's domain of empirical adequacy with its domain of application.

\subsection{Instrumental Reliability}

In a previous paper I introduced the notion of instrumental reliability and I argued that this notion should be distinguished very carefully from the more common notion of empirical adequacy. ${ }^{23}$ The notion of empirical confirmation ought to be correspondingly separated from that which I called degree of confidence. I hope this paper contributes to a more complete understanding of that claim. The instrumental reliability of a theory is linked to its effectiveness as a tool in application. Roughly, all other things being equal, the larger a theory's domain of application, the higher the theory's instrumental reliability. However, as we have seen, a theory's domain of application is never smaller than its domain of empirical adequacy, and will typically be much larger. Therefore if the notion of instrumental reliability is to capture the various ways in which scientific theories are applied, it must be kept distinct from (any sensible notion of) empirical adequacy.

Let me recapitulate briefly why. The case study in superconductivity points to the existence of a variety of ways in which scientific theories are applied. Some scientific applications are embeddable into theory à la van Fraassen; others are reductions in the sense of Friedman. I argue that there is a further class of applications that are not reducible to theory, nor can they be embedded in theory. The London model does not raise the degree of confirmation of electromagnetic theory; it raises its degree of confidence - that is, it gives us a reason to believe that the theory is instrumentally reliable, i.e. that it will go on to provide successful applications. The scientific community's commitment to classical electromagnetism in the face of superconducting phenomena seems to have required neither the belief that the theory was true, nor that it was empirically adequate. Only a strong sense of confidence that the theory could be successfully applied was involved. ${ }^{24}$

${ }^{23}$ See Suárez 1999.

${ }^{24}$ Thus Dahl (1992, p. 164) claims that the scientific community displayed a "dogmatic faith" in the applicability to superconductivity of both thermodynamics and standard electromagnetic theory. 
Scientific realism and constructive empiricism share a common core, which goes beyond what instrumental reliability requires. On either view, a minimum requirement for the acceptance of a scientific theory is that the theory must be empirically adequate -i.e. that what the theory states about the phenomena must indeed be the case. The constructive empiricist argues that the acceptance of a theory need only involve the belief that it is empirically adequate. Theories may have other virtues besides empirical adequacy — such as simplicity, explanatory power, aesthetic value, or even the virtue of being true - but belief in the theory's empirical adequacy is the only doxastic attitude required for the acceptance of the theory. By contrast, the realist argues that the belief that a theory is true, or likely to be true, and not just empirically adequate, is also required for its acceptance. For the realist a good theory, in addition to being empirically adequate, should also be true, or likely to be true - not only true to the phenomena, but true tout court, true to the world.

The instrumental reliability of a theory on the other hand provides neither grounds to believe that the theory is true, nor that it is empirically adequate - it points neither towards scientific realism nor towards constructive empiricism. The kind of confidence that the instrumental reliability of the theory inspires entails nothing per se regarding its empirical adequacy. As Imre Lakatos used to argue, a false and falsified theory might go on to produce multiple applications, at least for a good while. Or in my terminology: an empirically inadequate theory may nevertheless exhibit great instrumental reliability. While truth and empirical adequacy are epistemic virtues of theories, instrumental reliability is merely a pragmatic one.

\subsection{The Application of Scientific Theories}

It should thus be clear that I am not advocating the first horn of my dilemma; unlike Bueno I do not advocate (not on the basis of this case study, at any rate) a revision of any of our entrenched concepts of empirical adequacy and empirical confirmation. There are substantial issues to be settled between van Fraassen's representationalism and Friedman's reductionism as to what empirical confirmation gives actual credence for - whether it gives reason to believe in the unobservable structure of our theories, or only the observable one. It is possible that representationalism is more appropriate to understand the relationship between theory and phenomena required for empirical adequacy in some areas of physics, and reduction for others. My 
claim is that in either case, the domain of empirical adequacy will not cover the typically much larger domain of application.

An antecedent of this claim is Duhem's distinction between experiments of application and experiments of testing, which I reviewed in section 1. There, application and confirmation diverge too. So why should they overlap in general? Application is after all also distinct from explanation, and this latter claim is entrenched. It is precisely this part of Bas van Fraassen's work that has received wider acceptance, in the form of the pragmatic theory of explanation. Van Fraassen has perhaps done more than anyone in order to neatly separate the epistemic norm of empirical adequacy (and confirmation) from the pragmatic one of explanation. Consider for instance the well known arguments in chapter 5 of The Scientific Im$a_{g e} e^{25}$ in favour of separating explanation from confirmation. Many of them carry over to support my case, and can be turned into arguments that separate explanation from application as well. A couple of other famous books arguing for the separation of explanatory power and confirmation are Nancy Cartwright's How the Laws of Physics Lie and Ian Hacking's Representing and Intervening. ${ }^{26}$ Similarly Arthur Fine and others have argued that quantum theory can be applied to the Einstein-Podolsky-Rosen correlations without explaining them. ${ }^{27}$ More recently, Henk de Regt and Dennis Dieks have also argued in favour of a pragmatic norm of explanatory "understanding" - distinct and additional to application - which they argue is just as essential an aim of science. ${ }^{28}$

My concerns to separate application from confirmation in many ways mirror all these attempts to separate application from explanation. But where all these authors tend to see only two distinct norms (explanation, and confirmation), I see three (explanation, confirmation, and application), for I claim the latter two are also distinct; we should not in general mistake the domain of application of a theory for its domain of empirical adequacy. The case study in superconductivity illustrates one way in which theory helps to provide a description of a phenomenon from which it cannot receive con-

${ }^{25}$ Van Fraassen 1980, chapter 5.

${ }^{26}$ Cartwright 1983, Hacking 1983. A very explicit defence of this view is Nancy Cartwright's little-known 1989 paper, entitled: "The Born-Einstein Debate: Where application and explanation separate".

${ }^{27}$ See the essays by Fine, van Fraassen and others in Cushing and McMullin 1989.

${ }^{28}$ De Regt and Dieks 2005, esp. p. 143. 
firmation. Explanation, confirmation and application are all distinct norms, in possibly decreasing order of strength. ${ }^{29}$

\section{REFERENCES}

Bogen, J. and J. Woodward, 1988, "Saving the Phenomena", The Philosophical Review, vol. 97, pp. 303-352.

Bueno, O., 1997, "Empirical Adequacy: A Partial Structures Approach", Studies in History and Philosophy of Science, vol. 28, pp. 585-610.

Cartwright, N., 1999a, "Models and the Limits of Theory: Quantum Hamiltonians and the BCS Model of Superconductivity", in Morgan and Morrison 1999, pp. 241-281.

- 1999b, The Dappled World: A Study of the Boundaries of Science, Cambridge University Press, Cambridge.

— 1989, "The Born-Einstein Debate: Where Application and Explanation Separate", Synthese, vol. 81, pp. 271-282.

Oxford.

Cartwright, N., T. Shomar and M. Suárez, 1995, "The Toolbox of Science", in Herfel et al. (eds.), Theories and Models of Scientific Processes, Poznan Studies in the Philosophy of the Sciences and the Humanities, Rodopi, Amsterdam.

Churchland, P.M. and C.A. Hooker, Images of Science. Essays on Realism and Empiricism with a Reply form Bas C. van Fraassen, The University of Chicago Press, Chicago.

Cushing, J. and E. McMullin (eds.), 1989, Philosophical Consequences of Quantum Theory, University of Notre Dame Press, Notre Dame.

Da Costa, N. and S. French, 1990, "The Model Theoretic Approach in the Philosophy of Science", Philosophy of Science, vol. 57, pp. 248-265.

Dahl, P.F., 1992, Superconductivity: Its Historical Roots and Development from Mercury to the Ceramic Oxides, American Institute of Physics, New York.

${ }^{29}$ This paper has a distant ancestor in chapter 2 of my PhD thesis (Suárez 1997), which in turn was an improved version of a manuscript that I circulated in 1994 under the title "How Theories Save Phenomena: A Case Against Embedding". I became interested in different topics after I was awarded my PhD, so the paper got shelved indefinitely (although Suárez 1999 contains a discussion of instrumental reliability and related results). I would like to thank all those people who offered their comments and suggestions during the years 1994-1997, as well as Germán Guerrero Pino, whose spirited defence of his own PhD Thesis (Guerrero Pino 2003) prompted me to return to the topic, Enrique Casanova for his helpful feedback on the issues in section 3 , and an anonymous referee of this journal for detailed comments. 
Dalla Chiara, M. and G. Toraldo di Francia, 1979, "Formal Analysis of Empirical Theories", in Problems in the Foundations of Physics, Proceedings of the International School of Physics "Enrico Fermi".

Demopoulos, W., 2003, "On the Rational Reconstruction of Our Theoretical Knowledge", British Journal for the Philosophy of Science, vol. 54, no. 3, pp. 371-403.

De Regt, H. and D. Dieks, 2005, "A Contextual Approach to Scientific Understanding”, Synthese, vol. 144, pp. 137-170.

Duhem, P., 1969, To Save the Phenomena, The University of Chicago Press, Chicago.

- 1954, The Aim and Structure of Physical Theory, Princeton University Press, Princeton.

French, S. and J. Ladyman, 1997, "Superconductivity and Structures: Revisiting the London Account", Studies in the History and Philosophy of Modern Physics, vol. 28, no. 3, pp. 363-393.

Friedman, M., 1982, Review of "Bas C. van Fraassen, The Scientific Image (1980)", Journal of Philosophy, vol. 79, no. 5, pp. 274-283.

Giere, R., 1988, Explaining Science: A Cognitive Approach, The University of Chicago Press, Chicago.

Guerrero Pino, G., 2003, Enfoque semántico de las teorías. Estructuralismo y espacio de estados: coincidencias y divergencias, $\mathrm{PhD}$ thesis, Universidad Complutense de Madrid.

Hacking, I., 1983, Representing and Intervening, Cambridge University Press, Cambridge.

Kaiser, M., 1991, "From Rocks to Graphs: The Shaping of Phenomena", Synthese, vol. 89, pp. 111-133.

Mayo, D., 1996, Error and the Growth of Experimental Knowledge, The University of Chicago Press, Chicago.

Morgan, M. and M. Morrison (eds.), 1999, Models as Mediators, Cambridge University Press, Cambridge/New York.

Morrison, M., 1990, "Unification, Realism and Inference", British Journal for the Philosophy of Science, vol. 41, pp. 305-332.

Nagel, E., 1979, The Structure of Science, Hackett, Indianapolis.

Prezelecki, M., 1969, The Logic of Empirical Theories, Routledge, London.

Suárez, M., 2004, "Causal Processes and Propensities in Quantum Mechanics", Theoria, vol. 19, no. 3, pp. 271-300.

- 1999, "The Role of Models in the Application of Scientific Theories: Epistemological Implications", in Morgan and Morrison 1999, pp. 168196.

, 1997, Models of the World, Data-Models and the Practice of Science: The Semantics of Quantum Theory, PhD thesis, London School of Economics.

Suppe, F., 1974, The Structure of Scientific Theories, University of Illinois Press, Urbana. 
Suppes, P., 2002, Representation and Invariance of Scientific Structures, CSLI, Stanford.

- 1970, Set-Theoretical Structures in Science, Tech. Report, Institute for Mathematical Studies in the Social Sciences, Stanford University. (Reprinted as Suppes 2002.)

Suppes, P., 1969, "Models of Data", in Studies in the Methodology and Foundations of Science, Reidel, Dordrecht, pp. 24-35.

— , 1961, "A Comparison of the Meaning and Use of Models in Mathematic and the Empirical Sciences", in H. Freudenthal (ed.), The Concept and Role of the Model in Mathematics and the Natural and Social Sciences, Reidel, Dordrecht, pp. 163-177.

Van Fraassen, B., 1989, Laws and Symmetry, Oxford University Press, Oxford.

Van Fraassen, B., 1985, "Empiricism in the Philosophy of Science", in Churchland and Hooker (eds.), pp. 245-305.

- 1982, "The Charybdis of Realism: Epistemological Implications of Bell's Inequality", Synthese, vol. 52, pp. 25-38. (Reprinted in Cushing and McMullin 1989.)

— 1980, The Scientific Image, Oxford University Press, Oxford.

Wojcicki, R., 1974, "Set-Theoretical Representations of Empirical Phenomena", Journal of Philosophical Logic, vol. 3, pp. 337-343.

Worrall, J., 1984, "An Unreal Image", British Journal for the Philosophy of Science, vol. 35, pp. 65-79.

Received October 24, 2003; revised October 19, 2004; accepted January 19, 2005. 